\title{
Ultra-Fast Layer-by-Layer Approach for Depositing Flame Retardant Coatings on Flexible PU Foams within Seconds
}

\author{
F. Carosio ${ }^{* \dagger}$ and J. Alongi \\ ${ }^{\dagger}$ Dipartimento di Scienza Applicata e Tecnologia, Politecnico di Torino, Alessandria campus, Viale Teresa Michel 5, 15121 \\ Alessandria, Italy \\ "Dipartimento di Chimica, Università degli Studi di Milano, Via Golgi 19, 20133 Milano, Italy
}

Supporting Information

ABSTRACT: In this letter, we are presenting a novel approach for the deposition of layer-by-layer (LbL) coatings capable of conferring flame retardant properties to flexible polyurethane foams exploiting subsecond deposition times. The process yields nanoscale coatings able to reduce by $33 \%$ one of the main fire safety parameters, namely the heat release rate peak, with a total treatment time of only $2.5 \mathrm{~s}$. This new approach turned out to be three to 4 orders of magnitude faster than conventional LbL treatments. Such results make it possible for the exploit of $\mathrm{LbL}$ as a competitive, efficient and ecofriendly technology at industrial scale.

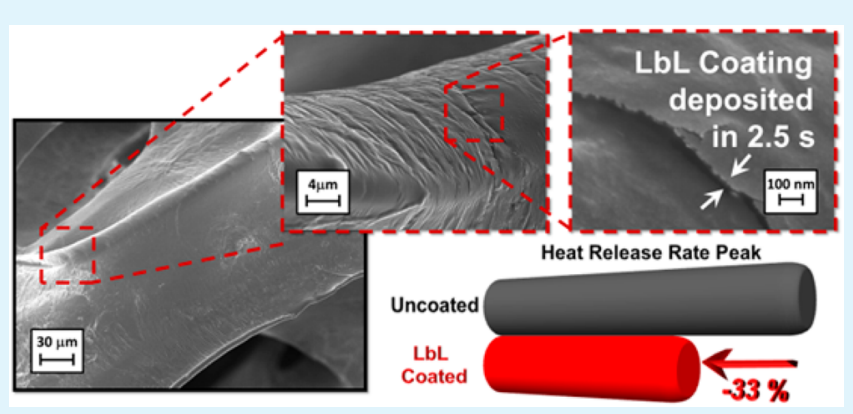

KEYWORDS: layer by layer, chitosan, fast deposition, polyurethane foams, flame retardancy, cone calorimetry

T $\mathrm{n}$ the past decade, the LbL has arisen as standard for the controlled fabrication of nanostructured coatings or surfacebased devices. ${ }^{1,2}$ The reason for such success relies in the intrinsic simplicity of this technique that exploits the physical and chemical interactions occurring in solution between two or more reagents for the step-by-step deposition of stratified or highly interpenetrated nanostructured coatings. ${ }^{3}$ Because the most widely exploited interaction is represented by the electrostatic attraction between water-based suspensions of nanoparticles or polyelectrolytes, for virtually any aqueousbased system it is possible to find a matching counterpart and deposition conditions, yielding a steady film build-up. Furthermore, the assembly final properties can be controlled by means of key parameters like molecular weight, temperature, ionic strength, and $\mathrm{pH}$. $^{1}$

Recently, the LbL assembly has been adopted for the buildup of fire protective coatings, demonstrating how nanostructured assemblies can be efficient in the protection of fabrics, foams and plastics. $^{4-7}$ Different functional coatings comprising of nanoparticles, polyelectrolytes and biomacromolecules can be deposited. ${ }^{8-12}$ From a practical and real point of view, the LbL assembly of fire protective coatings represents one of the possible answer to the current and incessant demand for green and nontoxic solutions since the some of the currently adopted chemicals have been proven to be toxic and persistent in the environment, eventually ending in the food chain, and in the bodies of animals and humans. ${ }^{13}$ Thus, it is easy to understand why the LbL with the use of aqueous-based solutions at low concentration, the possibility of employing green components and, most important, of achieving extraordinary results has attracted the interest of both academic and industrial researchers. Unfortunately, this technology is currently held back by the enormous (if compared to industrial scale) amount of time often required to obtain the desired functional coatings. An additional drawback is represented by need to remove a large amount of water from the PU structure after each deposition/rinsing step.

Spray-assisted LbL deposition is often proposed as a viable tool in order to speed up the process; ${ }^{14-16}$ however, this approach is of limited practicability when considering a porous substrate such as flexible open cell polyurethane (PU) foams. Indeed, for this latter substrate a sprayed polyelectrolyte/ nanoparticle solution would not be able to fully penetrate inside the PU complex 3D structure and coat each available surface (as thicknesses are in the range of $10-50 \mathrm{~mm}$ ), resulting in an incomplete and thus inefficient coating; in addition, the porous structure may also result difficult to rinse. For these reasons, dipping appears to be the best way to achieve a homogeneous and efficient nanocoating on PU foams. Unfortunately, although recent research solutions not involving spray have provided strong improvements in reducing the duration or the number of the deposition steps required, ${ }^{17-20}$ the standard treatment time still remains on the order of several minutes, whereas the water removal issue has never been addressed: unacceptable constraints for a possible real industrial exploitation.

\footnotetext{
Received: January 16, 2016

Accepted: February 29, 2016

Published: February 29, 2016
} 


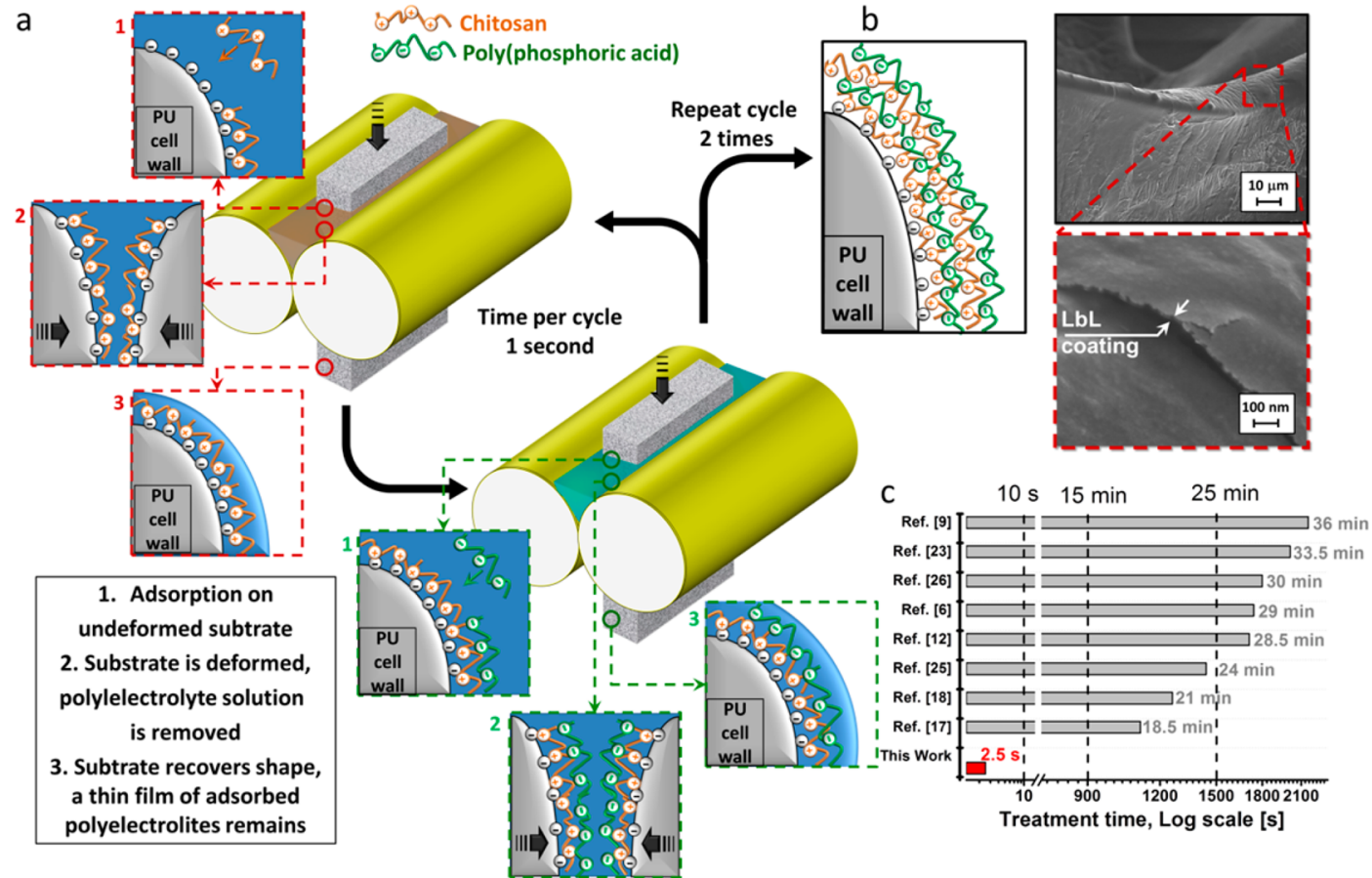

Figure 1. Fast LbL deposition on flexible PU foams: (a) schematization of the process and phenomena occurring during the LbL assembly on flexible PU foam, (b) schematization of a 2 bilayers (BL) dried coating and FESEM pictures of $2 \mathrm{BL}$ treated foam, (c) comparison between overall treatment times reported in the scientific literature for $\mathrm{LbL}$ on PU foams and in this work.

Thus, finding a way for the fast deposition of functional LbL coatings represents a crucial issue to be addressed for the real life application and industrial applications of this technology.

In this paper, we are proposing a novel approach for the deposition of LbL coatings capable of conferring fire protection properties to PU exploiting sub second deposition times which are three to 4 orders of magnitude faster than standard and current LbL treatments. This approach is based on the standard $\mathrm{LbL}$ adsorption process that relies on the interactions between oppositely charged species, significantly accelerating the deposition times from some minutes to sub seconds by imparting strong deformation to the substrate, as schematically depicted in Figure 1.

As reported in Figure 1 a, the substrate is fist immerged in the solution and then quickly deformed in order to remove the excess of polyelectrolyte/water before moving to the next deposition step. This process practically solves the two main problems related to $\mathrm{LbL}$ and $\mathrm{PU}$ foams as it allows for extremely short deposition times and an almost instantaneous, reproducible and efficient removal of excess solution. In the adopted configuration, the deposition time (i.e., the time the substrate is put into contact with the solution) has been set at $0.5 \mathrm{~s}$ with a total treatment time of only $2.5 \mathrm{~s}$ (including a first deposition step for surface activation and assuming a continuous process) for the deposition of functional $2 \mathrm{BL}$ coatings. This is in strong contrast with previously reported LbL coatings where the total treatment times (calculated as the sum of the reported deposition times under the assumption of assuming a continuous process) are in the order of tens of minutes, as reported in Figure 1. Despite the fact that the total treatment time may be reduced by several orders of magnitude, the proposed approach poses several inherent scientific challenges that must be faced such as the use of a high efficient $\mathrm{LbL}$ formulation capable of reaching high performances at low deposition steps, while withstanding the deformation imparted to the sample during the process. Indeed, when the substrate is compressed and its volume is reduced to the minimum, the very same deformation is transferred to the coating that can either survive the deformation or detach from the surface. To overcome such challenges, we propose the use of an allpolyelectrolyte $\mathrm{LbL}$ assembly specifically designed for imparting more flexibility to the coating during the process with respect to a nanoparticles containing one. Indeed, the use of nanoparticles has been proven to imply extra constraints on the LbL assembly conditions because of their propensity for self-aggregation and their rigid nature eventually increasing the stiffness of the assembled nanocoating. ${ }^{21,22}$

This latter choice represents an additional challenge, as up to now the presence of nanoparticles in the coating has been referred as a mandatory condition in order to obtain the desired fire protection performances while keeping low the number of deposition steps. ${ }^{23-26}$ For the above reasons, in this work we address the use of chitosan $(\mathrm{CH})$ and poly(phosphoric acid) (PPA) specifically chosen in order to minimize the layers needed for a functional coating; this formulation represents a LbL system in which thermally stable structures can be generated, due to the interaction of $\mathrm{CH}$ and PPA upon heating as demonstrated for similar $\mathrm{LbL}$ coatings containing $\mathrm{CH}$ and ammonium polyphosphate. ${ }^{27}$ Such structures can act as a physical barrier and protect the PU from heat, mass, and oxygen transfer, thus resulting in a fire protection.

First, the LbL growth of the selected constituents has been checked by infrared spectroscopy on silicon wafers. The coating has been deposited on PU foams a lab-scale padder, following the procedure graphically described in Figure 1 a. The morphology of the untreated and 2 BL-treated has been then imaged by FESEM microscopy and the achieved flame retardant properties have been evaluated by means of cone calorimetry under irradiative heat flux of $35 \mathrm{kw} / \mathrm{m}^{2}$. 


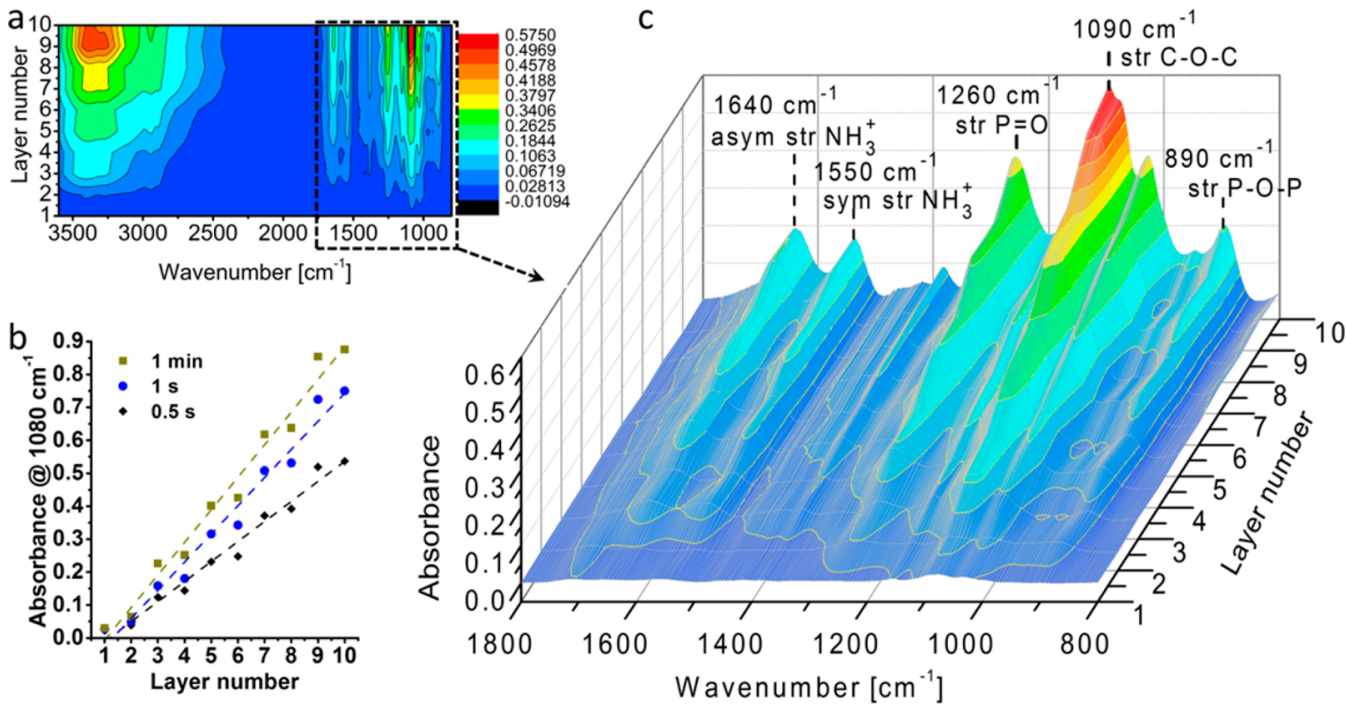

Figure 2. Coating growth followed by IR spectroscopy: (a) intensity surface plot as a function of each deposited layer (odd and even numbers corresponding to chitosan and poly(phosphoric acid) adsorption steps, respectively), (b) layer-dependent intensity of the peak at $1080 \mathrm{~cm}^{-1}$ and (c) $3 \mathrm{D}$ projection of the $1800-800 \mathrm{~cm}^{-1}$ IR region.
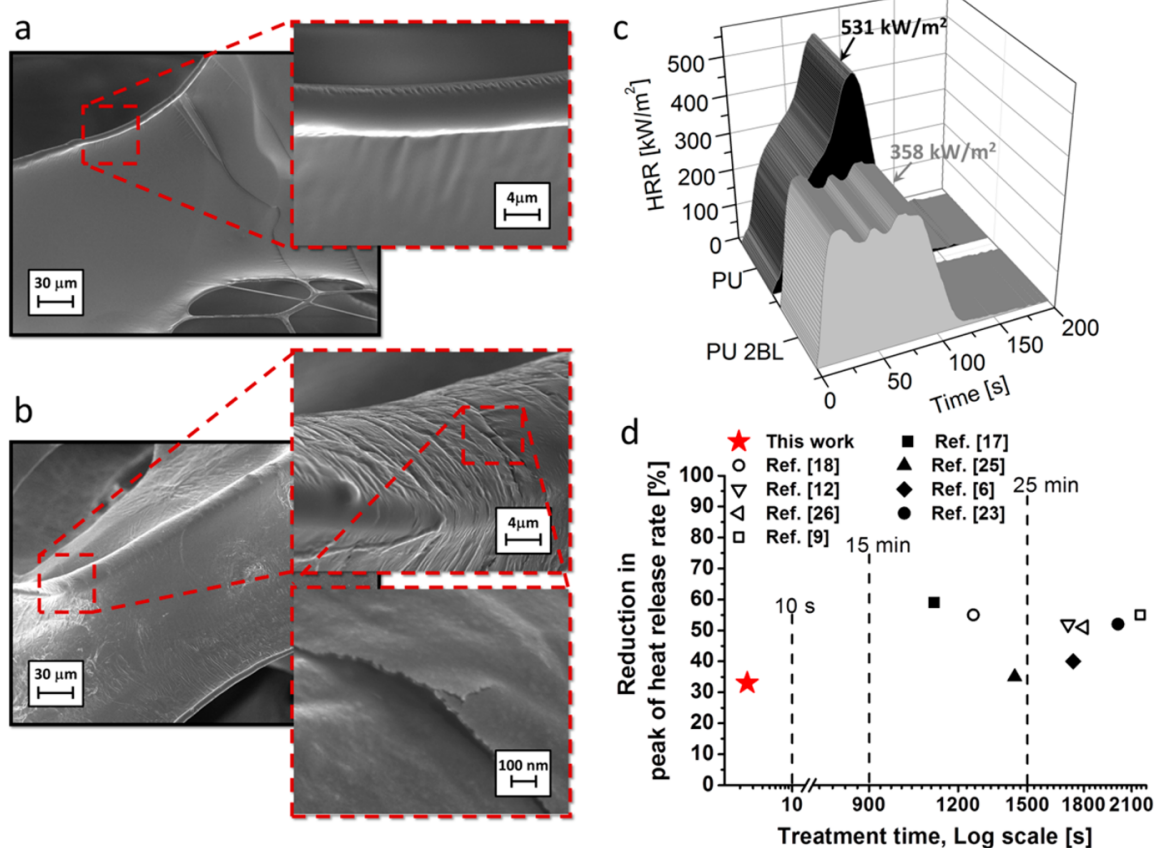

Figure 3. FESEM observations performed on (a) unmodified and (b) 2 BL-treated PU foam (inlets show detailed surface region at increased magnification), (c) heat release rate 3D plots of unmodified and 2 BL-modified PU foam, (d) comparison between the achieved reduction in peak of heat release rate as a function of the deposition time for this work and other works published in the scientific literature.

Assemblies have been grown on $\mathrm{Si}$ wafer employing the conventional dipping time of 1 min per layer and, subsequently, reducing it to very short deposition times in order to mimic the deposition conditions adopted for the flexible PU foams. The main results are reported in Figure 2.

As depicted in Figure 2, the characteristics signals of $\mathrm{CH}$ and PPA increase in intensity after each deposition step. As far as chitosan is concerned, $\mathrm{NH}_{3}{ }^{+}$asymmetric and symmetric stretching vibrations and glyosidic linkage $\mathrm{C}-\mathrm{O}-\mathrm{C}$ stretching vibrations can be found at 1640, 1550, and $1080 \mathrm{~cm}^{-1}$, respectively; on the other hand, PPA signals related to stretching of $\mathrm{P}=\mathrm{O}, \mathrm{PO}_{2}{ }^{-}$, and $\mathrm{P}-\mathrm{O}-\mathrm{P}$ are easily detected at 1260 and $890 \mathrm{~cm}^{-1}$. The intensity plot of the $1080 \mathrm{~cm}^{-1}$ peak, reported in Figure $2 b$, suggests a linear growth regime for this assembly either at $1 \mathrm{~min}$ or 1 or $0.5 \mathrm{~s}$. As expected, by reducing adsorption time to $0.5 \mathrm{~s}$, the intensity at each deposition step decreases; this behavior was expected as the reduction in deposition time would allow for a lower quantity of polyelectrolyte to be adsorbed on the surface. Nevertheless, such reduced linear growth demonstrates that the LbL deposition of selected reagents can occur even with extremely low deposition times.

Then the coating was easily transferred to open cell PU foams through the procedure described in Figure 1. Samples were imaged before and after the deposition and the resulting 
flame retardant properties evaluated by cone calorimetry; collected data are reported in Figure 3.

When imaged at low magnification, the unmodified PU foam shows a typical 3D structure made of open cells connected to each other (Figure S1) at high magnifications, the surface of the cell wall is revealed and appears smooth and clean (see Figure $3 \mathrm{a}$ and its inlet). The deposition of $2 \mathrm{BL}$ is capable of imparting apparent changes in foam surface morphology yielding a thin and homogeneous nanocoating that uniformly coats the PU 3D structure, easily following its complex geometry and without altering its open cell nature (compare Figure S1a and b). In addition, as reported in the highest-magnification micrographs (inlet of Figure $3 \mathrm{~b}$ ), this all-polymer coating has been found wrapped around the cell wall edges, thus demonstrating that the assembly survived the deposition process and the related compression deformations without the formation of polyelectrolyte complex aggregates. Elemental analyses (Figure S2) confirmed the presence of both components within the coating.

The functional properties of the deposited coating have been assessed by means of cone calorimetry. This test evaluates the specimen reactions when exposed to a heat flux of $35 \mathrm{~kW} / \mathrm{m}^{2}$, typically found in developing fires. The high temperatures reached favor the sample degradation, release combustible volatile gases and flaming combustion. During the test, the heat release rate (HRR) of the sample is evaluate and plotted as a function of time (as reported in Figure 3c); from the plot, the maximum peak is calculated (pkHRR) and used as main parameter for performance evaluation. Other cone parameters are reported in Table $S 1$.

Upon exposure to the cone heat flux, the structure of the unmodified PU quickly collapses eventually forming a vigorously burning pool of a low viscosity liquid and reaching the maximum in heat release rate $\left(531 \mathrm{~kW} / \mathrm{m}^{2}\right)$. Such behavior is well-known in the literature and has been related to an increase in the combustion rate of other burning items and, subsequently, a faster fire spread. ${ }^{29,30}$

This phenomenon does not occur when the PU is treated with $2 \mathrm{BL}$ of $\mathrm{CH} / \mathrm{PPA}$; instead of collapsing, the structure, protected by the thermally stable carbonaceous structures produced by the coating, slowly shrinks as it is slowly consumed by the fire, thus resulting in a $33 \%$ reduction in pKHRR $\left(358 \mathrm{~kW} / \mathrm{m}^{2}\right)$. As showed in Figure 3d, this result is similar to other LbL assemblies published in the literature, even if in the present manuscript the number of layers is significantly lower than other systems. Furthermore, the fact that it took only $2.5 \mathrm{~s}$ to achieve such performances is extremely crucial and in strong contrast with other works, where more than $15 \mathrm{~min}$ are required to achieve similar results.

In conclusion, in the present work we have presented an ultrafast approach for the deposition of functional and environmentally friendly LbL coatings on flexible substrates. Morphological assessment proved the effective deposition of a LbL nanocoating on flexible PU foams while combustion tests demonstrated the efficiency of the deposited coatings in reducing one of the main parameters in fire safety, the peak of heat release rate. This work makes it possible for the use of layer by layer as a competitive and efficient technology to be exploited at industrial scale, not only for the production of fire protecting coatings but also for other application fields employing flexible substrates.

\section{ASSOCIATED CONTENT}

\section{Supporting Information}

The Supporting Information is available free of charge on the ACS Publications website at DOI: 10.1021/acsami.6b00598.

Full experimental details, low-magnification FESEM micrographs of untreated and 2BL-treated PU foams, elemental analysis performed on 2 BL-treated PU foam, and cone calorimetry data of untreated and LbL-treated PU foams (PDF)

\section{AUTHOR INFORMATION}

\section{Corresponding Author}

*E-mail: federico.carosio@polito.it. Tel.: +39 0131229303. Fax: +390131229399.

\section{Notes}

The authors declare no competing financial interest.

\section{ACKNOWLEDGMENTS}

The authors want to thank Mr. Mauro Raimondo for FE-SEM analyses and Mr. Fabio Cuttica for cone calorimetry tests.

\section{REFERENCES}

(1) Decher, G.; Schlenoff, J. B. Multilayer Thin Films: Sequential Assembly of Nanocomposite Materials, 2nd ed.; Wiley-VCH: Weinheim, Germany, 2012.

(2) Decher, G. Fuzzy Nanoassemblies: Toward Layered Polymeric Multicomposites. Science 1997, 277, 1232-1237.

(3) Hammond, P. T. Form and Function in Multilayer Assembly: New Applications at the Nanoscale. Adv. Mater. 2004, 16, 1271-1293.

(4) Malucelli, G.; Carosio, F.; Alongi, J.; Fina, A.; Frache, A.; Camino, G. Materials Engineering for Surface-Confined Flame Retardancy. Mater. Sci. Eng., R 2014, 84, 1-20.

(5) Li, Y. C.; Schulz, J.; Mannen, S.; Delhom, C.; Condon, B.; Chang, S.; Zammarano, M.; Grunlan, J. C. Flame Retardant Behavior of Polyelectrolyte-Clay Thin Film Assemblies on Cotton Fabric. ACS Nano 2010, 4, 3325-3337.

(6) Kim, Y. S.; Davis, R.; Cain, A. A.; Grunlan, J. C. Development of Layer-by-Layer Assembled Carbon Nanofiber-Filled Coatings to Reduce Polyurethane Foam Flammability. Polymer 2011, 52, 28472855.

(7) Apaydin, K.; Laachachi, A.; Ball, V.; Jimenez, M.; Bourbigot, S.; Toniazzo, V.; Ruch, D. Polyallylamine-Montmorillonite as Super Flame Retardant Coating Assemblies by Layer-by-Layer Deposition on Polyamide. Polym. Degrad. Stab. 2013, 98, 627-634.

(8) Laufer, G.; Carosio, F.; Martinez, R.; Camino, G.; Grunlan, J. C. Growth and Fire Resistance of Colloidal Silica-Polyelectrolyte Thin Film Assemblies. J. Colloid Interface Sci. 2011, 356, 69-77.

(9) Carosio, F.; Di Blasio, A.; Cuttica, F.; Alongi, J.; Malucelli, G. Self-assembled Hybrid Nanoarchitectures Deposited on Poly(urethane) Foams Capable of Chemically Adapting to Extreme Heat. RSC Adv. 2014, 4, 16674-16680.

(10) Li, Y.-C.; Mannen, S.; Morgan, A.; Chang, S.; Yang, Y.; Condon, B.; Grunlan, J. C. Intumescent All-Polymer Multilayer Nanocoating Capable of Extinguishing Flame on Fabric. Adv. Mater. 2011, 23, 3926-3931.

(11) Carosio, F.; Di Blasio, A.; Alongi, J.; Malucelli, G. Green DNABased Flame Retardant Coatings Assembled Through Layer by Layer. Polymer 2013, 54, 5148-5153.

(12) Laufer, G.; Kirkland, C.; Morgan, A. B.; Grunlan, J. C. Exceptionally Flame Retardant Sulfur-Based Multilayer Nanocoating for Polyurethane Prepared from Aqueous Polyelectrolyte Solutions. ACS Macro Lett. 2013, 2, 361-365.

(13) Stieger, G.; Scheringer, M.; Ng, C. A.; Hungerbuhler, K. Assessing the Persistence, Bioaccumulation Potential and Toxicity of Brominated Flame Retardants: Data Availability and Quality for 36 
Alternative Brominated Flame Retardants. Chemosphere 2014, 116, $118-123$.

(14) Carosio, F.; Di Blasio, A.; Cuttica, F.; Alongi, J.; Frache, A.; Malucelli, G. Flame Retardancy of Polyester Fabrics Treated by SprayAssisted Layer-by-Layer Silica Architectures. Ind. Eng. Chem. Res. 2013, 52, 9544-9550.

(15) Izquierdo, A.; Ono, S. S.; Voegel, J. C.; Schaaf, P.; Decher, G. Dipping Versus Spraying: Exploring the Deposition Conditions for Speeding up Layer-by-Layer Assembly. Langmuir 2005, 21, 75587567.

(16) Schaaf, P.; Voegel, J. C.; Jierry, L.; Boulmedais, F. Spray-Assisted Polyelectrolyte Multilayer Buildup: from Step-by-Step to Single-Step Polyelectrolyte Film Constructions. Adv. Mater. 2012, 24, 1001-1016.

(17) Cain, A. A.; Plummer, M. G. B.; Murray, S. E.; Bolling, L.; Regev, O.; Grunlan, J. C. Iron-Containing, High Aspect Ratio Clay as Nanoarmor That Imparts Substantial Thermal/Flame Protection to Polyurethane with a Single Electrostatically-Deposited Bilayer. J. Mater. Chem. A 2014, 2, 17609-17617.

(18) Carosio, F.; Negrell-Guirao, C.; Alongi, J.; David, G.; Camino, G. All-polymer Layer by Layer Coating as Efficient Solution to Polyurethane Foam Flame Retardancy. Eur. Polym. J. 2015, 70, 94103.

(19) Carosio, F.; Fontaine, G.; Alongi, J.; Bourbigot, S. Starch-Based Layer by Layer Assembly: Efficient and Sustainable Approach to Cotton Fire Protection. ACS Appl. Mater. Interfaces 2015, 7, 1215812167.

(20) Carosio, F.; Alongi, J. Few Durable Layers Suppress Cotton Combustion Due to the Joint Combination of Layer by Layer Assembly and UV-Curing. RSC Adv. 2015, 5, 71482-71490.

(21) Lee, D.; Rubner, M. F.; Cohen, R. E. All-Nanoparticle ThinFilm Coatings. Nano Lett. 2006, 6, 2305-2312.

(22) DeRocher, J. P.; Mao, P.; Kim, J. Y.; Han, J.; Rubner, M. F.; Cohen, R. E. Layer-by-Layer Deposition of All-Nanoparticle Multilayers in Confined Geometries. ACS Appl. Mater. Interfaces 2012, 4, 391-396.

(23) Laufer, G.; Kirkland, C.; Cain, A. A.; Grunlan, J. C. ClayChitosan Nanobrick Walls: Completely Renewable Gas Barrier and Flame-Retardant Nanocoatings. ACS Appl. Mater. Interfaces 2012, 4, $1643-1649$.

(24) Li, Y. C.; Kim, Y. S.; Shields, J.; Davis, R. Controlling Polyurethane Foam Flammability and Mechanical Behaviour by Tailoring the Composition of Clay-Based Multilayer Nanocoatings.

J. Mater. Chem. A 2013, 1, 12987-12997.

(25) Kim, Y. S.; Davis, R. Multi-Walled Carbon Nanotube Layer-byLayer Coatings With a Trilayer Structure to Reduce Foam Flammability. Thin Solid Films 2014, 550, 184-189.

(26) Li, Y.-C.; Davis, R. D.; Shields, J.; Kim, Y. S.; Yang, Y.-H. DNABased Nanocomposite Biocoatings for Fire-Retarding Polyurethane Foam. Green Mater. 2014, 2, 144-152.

(27) Carosio, F.; Alongi, J.; Malucelli, G. Layer by Layer Ammonium Polyphosphate-Based Coatings for Flame Retardancy of PolyesterCotton Blends. Carbohydr. Polym. 2012, 88, 1460-1469.

(28) Socrates, G. Infrared and Raman Characteristic Group Frequencies; Wiley: New York, 2004.

(29) Lefebvre, J.; Bastin, B.; Le Bras, M.; Duquesne, S.; Ritter, C.; Paleja, R.; Poutch, F. Flame Spread of Flexible Polyurethane Foam: Comprehensive Study. Polym. Test. 2004, 23, 281-290.

(30) Zammarano, M.; Kramer, R. H.; Harris, R.; Ohlemiller, T. J.; Shields, J. R.; Rahatekar, S. S.; Lacerda, S.; Gilman, J. W. Flammability Reduction of Flexible Polyurethane Foams Via Carbon Nanofiber Network Formation. Polym. Adv. Technol. 2008, 19, 588-595. 